\title{
AN APPLICATION SPECIFIC WINDOWS MANAGEMENT INSTRUMENTATION ARCHITECTURE FOR IMPROVING PERFORMANCE ON WIRELESS NETWORK
}

\author{
${ }^{1}$ Lavanya Sankara Narayanan and ${ }^{2}$ Murali Baskaran Venugopal \\ ${ }^{1}$ Department of IT, Faculty of Information Technology, \\ Paavai Engineering College, Namakkal, Tamil Nadu, India \\ ${ }^{2}$ Department of CSE, Faculty of Computer Science and Engineering, \\ Dhirajlal Gandhi College of Technology, Salem, Tamil Nadu, India
}

Received 2013-09-06; Revised 2013-10-23; Accepted 2013-11-28

\begin{abstract}
Wireless Communications is one of the fastest growing segments in the communications industry. Wireless Network is the network that facilitates communication among two or more computers connected through the standard network protocols, without network cabling. Generally, the Wireless Network is termed as Wireless Local Area Networks (WLAN). A WLAN is a collection of network components connected by electromagnetic (radio) waves instead of cables. Each Wireless Network consists of a Network Provider, which provides the network connection to the user. With the help of this network provider, the client (user) can able to transfer the data to and from the server. In this process, if the speed between the client and the server does not match, then there may be the chance for data loss. Due to the data loss, the network performance will get reduced. To avoid these problems, we propose architecture for wireless network communication, in order to reduce the data loss and other problems and to increase the network performance. The proposed architecture can able to perform on both the TCP layer and UDP layer.
\end{abstract}

Keywords: Quality of Service, Windows Management Instrumentation, Communication Channel

\section{INTRODUCTION}

In this Internet world, Network plays an important role to communicate and share information among people. There exist several types of networks based on the purpose of serving the information. The major categories among those types are wireless networks and wired networks, based on the connection of computers. In Wired Networks, more effort has been taken to establish the setup through wires and this wired network serves in very limited area. In this study, the terminology about the wireless network has been discussed in order to avoid some problems found in this wired network communication and to increase the efficiency of wireless network.

\subsection{Wireless Network}

A wireless network is used for data communications to transmit and receive data over the air, with the help of wireless media such as radio frequency technology. Wireless network becomes popular in order to minimize the need for wired connections. Communication through Wireless Network provides many benefits such as portability, flexibility, lower installation cost and so on, for both the organizations and users. Wireless technologies cover a broad range of differing capabilities oriented toward different uses and needs.

A wireless LAN basically consists of the network backbone, data collection units, laptops, wireless access points, wireless cards and software to manage the

Corresponding Author: Lavanya Sankara Narayanan, Department of IT, Faculty of Information Technology, Paavai Engineering College, Namakkal, Tamil Nadu, India 
wireless network. These components collectively provide means for wireless communications.

A single access point can support a small group of users and can function within a range of less than 100 to several hundred feets. Access points allow the wireless computer to access the LAN resources, such as file servers or any other resources. It can be achieved by connecting the WLAN to a wired LAN.

\subsection{Need for Wireless Network}

If the user and their organization need mobility, portability and accessibility of data, then the wireless networking is used to achieve these benefits. Many organizations uses this wireless network to gain advantage over long distance without any network cable. Some of the areas using this network are Corporate Information Systems, Shops/Retail, Education, Medical/Hospital, Warehousing and so on. Some of the advantages that the wireless network offers are as follows:

- Networks can be placed or installed anywhere and can be relocated at anytime without the constraints of wired networks

- Users can communicate from anywhere and anytime with the help of e-mail, file sharing and web browsing

- Able to maintain real-time information

- Wireless communications in larger organizations reduces the cost and time required to cable the campuses

- Can be used to improve the quality

\subsection{Wireless Communication}

In wireless communication, as already mentioned, there is no need for wired connection. In wired networks, Ethernet card is used to connect with the hub, for communication. Instead of the wires, a wireless network starts their communication with the help of waves and antennas.

In any network communication, there exist two parties namely Client and Server associated with this network. The Client and Server are the two end hosts which can transmit and receive the information to and from. This kind of architecture is termed as Client-Server Architecture. For sending and receiving the information, both the sender and receiver consist of Transmitter and Receiver. Through the transmitter, the client and server can able to transmit the data and with the help of the receiver, they can receive the data.

In Fig. 1, the client and server consist of antenna, to send and receive the information. A refers that; client sends data to server through Transmitter whereas the server receives the data through Receiver. B refers that; server sends data to client and the client receives it, which is contradiction to A. Thus, both the client and server consist of Transmitter and receiver to send and receive the information.

In this kind of communication, there involves 5 processes such as follows:

- Identifying Server and Client

- Sending Data

- Receiving Data

- Sending Acknowledgement

- Receiving Acknowledgement

The first step is to identify the client and server by itself, through electromagnetic waves. Upon receiving the wave signal, they establish an invisible connection between them and the two parties are named as client and server.

Upon establishing the connection, the next process is to transmit the data. The data has been transmitted by organizing the data into small packets. These packets are transmitted in sequence to the receiver. The receiver, upon receiving the packet, can send the acknowledgement to the transmitter. The Acknowledgement is nothing but the surety of receiving the packet and it is ready to receive the next packet. Upon receiving the acknowledgement, the transmitter can transmit the next packet. Thus, the sender and receiver transmit the data by packets.

In Fig. 2, A and B are the two parties started to communicate with the help of the antenna:

- Identifying the two channels A and B through Antenna

- A sends data to B through Antenna

- $B$ receives the data

- $\mathrm{B}$, upon receiving the data, sends the acknowledgement to A

- A receives the acknowledgement

If the acknowledgement not received from the receiver, then the sender identify that there is a packet loss on the receiver side. This packet loss is termed as Data Loss. This kind of loss can be occurred more in wireless networks. The data loss can be occurred in number of ways such as attenuation, noise, delays and so on.

If the receiver tries to analyze for data loss, instead of receiving the data, more data will loss or the network delay may occur due to not receiving the data sequentially. This kind of loss can also be occurred due to the low capacity on the receiver compared to the sender. Due to data loss, there may be the possibility of collision between the client and server. Thus the network performance gets down. In order to overcome these problems, the methodology proposed in this study performs well to reduce the data loss and to improve the network performance. There some techniques to tackle this problem. But our proposed architecture proposes the solution much better and simple than existing 
architecture. Also, to demonstrate the performance of the proposed architecture, the experiment can be carried out on a service provider.

\subsection{Related Work}

Based on various parameters, the efficiency of data dissemination was improved in wireless network.

\subsection{Framework for Network Coding}

Considered a simple wireless network topology to illustrate how network coding can improve throughput and energy efficiency objectives beyond routing solutions. Then, they extended the network coding problem to general wireless networks in conjunction with scheduling-based Medium Access Control (MAC). For that purpose, they partitioned the nodes into disjoint sets of transmitters and receivers that result in conflict-free network realizations with minimum cost (e.g., power) assignments. They separately activated distinct network realizations using a time division mechanism Then, they specified the content of network flows through network coding and derive transmission schedules to optimize the throughput or energy measures. Next, they presented a method of constructing time-varying linear network codes that satisfy the wireless network properties. Also, they verified via numerical results the superior performance of network coding over routing in terms of throughput and energy efficiency.

\subsection{Quality of Service}

An adaptive fault tolerant Quality of Service (QoS) control algorithms based on hop-by-hop data delivery utilizing "source" and "path" redundancy was developed, with the goal to satisfy application QoS requirements while prolonging the lifetime of the sensor system (Chen et al., 2011). They developed a mathematical model for the lifetime of the sensor system as a function of system parameters including the "source" and "path" redundancy levels utilized. They discovered that there exists optimal "source" and "path" redundancy under which the lifetime of the system was maximized while satisfying application QoS requirements. Numerical data were presented and validated through extensive simulation, with physical interpretations given, to demonstrate the feasibility of the algorithm design.

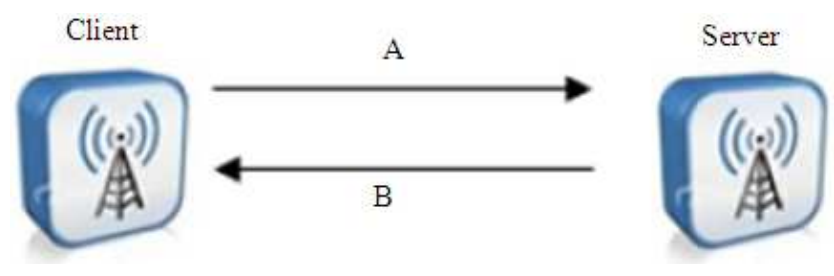

Fig. 1. Transmission between client and server
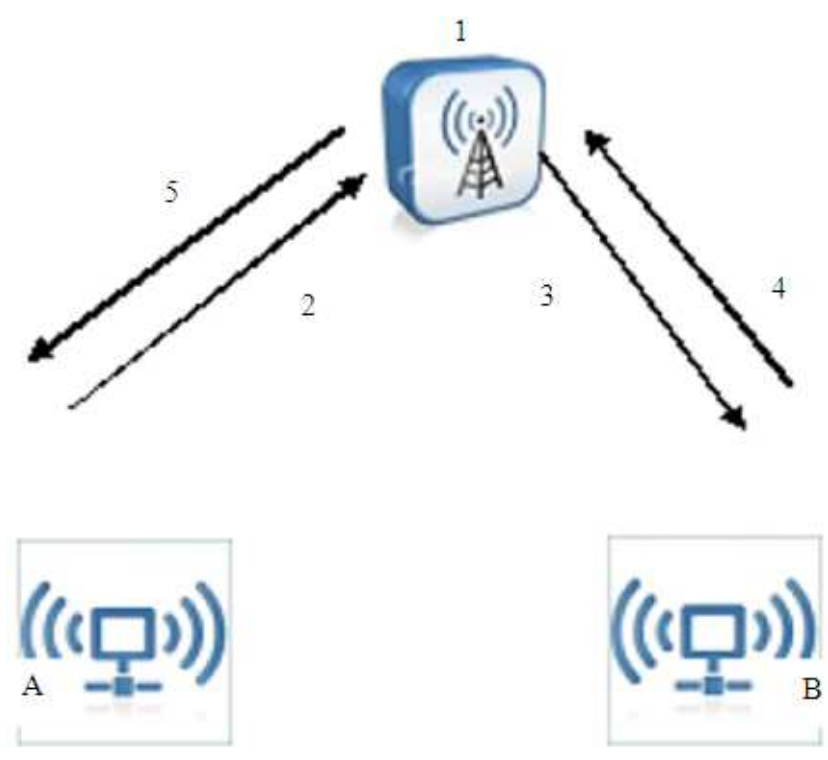

Fig. 2. Data Transmission using antenna between two channels 


\subsection{Asynchronous Transmission}

In wireless sensor network, the nodes were static. Nevertheless, node connectivity was subject to changes because of disruptions in wireless communication, transmission power changes (Cohen and Kapchits, 2011), or loss of synchronization between neighboring nodes. Hence, even after a sensor was aware of its immediate neighbors, it must continuously maintain its view, a process they called continuous neighbor discovery. In the study they distinguished between neighbor discovery during sensor network initialization and continuous neighbor discovery. They focused on the latter and view it as a joint task of all the nodes in every connected segment. Each sensor employs a simple protocol in a coordinate effort to reduce power consumption without increasing the time required to detect hidden sensors.

\subsection{Effect of Jammer Nodes}

How user data rate and the jamming effect were related was discussed and analyzed based on modulation schemes (Dhandhukia and Parmar, 2012). Transmitter, receiver and Jammer nodes were created in simulation environment. Effect of jammer radiated power was also analyzed.

\subsection{Energy Consumption among Nodes}

A cluster-based communication protocol that uses a multi-hop communication mode between the clusterheads was introduced. The protocol aims to reduce and evenly distribute the energy consumption among the sensor nodes and cover a large area of interest (Merzoug and Boukerram, 2011). The simulations show that the proposed protocol was efficient in terms of energy consumption, maximization of the network lifetime, data delivery to the sink and scalability.

\subsection{Communication Protocol}

Most of the clustering-based protocols use a singlehop communication to send data from the cluster-heads to the base station (Merzoug and Boukerram, 2011). In fact, they assumed that all sensor nodes can communicate directly with each other or with the base station. It becomes impossible when the size of the area increases and based on protocol architecture the cluster nodes are decentralized (Merzoug and Boukerram, 2011). The energy efficiency was maintained using cluster based communication protocol (Bajaber and Awan, 2011).

In study (Banavar et al., 2012), a distributed detection problem over fading Gaussian multiple-access channels is considered. Sensors observe a phenomenon and transmit their observations to a fusion center using the amplify and forward scheme. The fusion center has multiple antennas with different channel models considered between the sensors and the fusion center and different cases of channel state information are assumed at the sensors. The performance is evaluated in terms of the error exponent for each of these cases, where the effect of multiple antennas at the fusion center is studied. When there is channel information at the sensors, the gain in error exponent due to having multiple antennas at the fusion center is shown to be limited to a factor of $8 / \pi$ for Rayleigh fading channels between the sensors and the fusion center and independent of the number of antennas at the fusion center. Simple practical schemes and numerical methods using semidefinite relaxation techniques are presented that utilize the limited possible gains available. Simulations are used to establish the accuracy of the results.

In study (Ciuonzo et al., 2012), they proposed a channel-aware binary-decision fusion over a shared Rayleigh flat-fading channel with multiple antennas at the Decision Fusion Center (DFC). They presented the optimal rule and derive sub-optimal fusion rules, as alternatives with improved numerical stability, reduced complexity and lower system knowledge required. The set of rules is derived following both "Decode-andFuse" and "Decode-then-Fuse" approaches. Simulation results for performances are presented both under Neyman-Pearson and Bayesian frameworks. The effect of multiple antennas at the DFC for the presented rules is analyzed, showing corresponding benefits and limitations. Also, the effect on performances as a function of the number of sensors is studied under a total power constraint.

In study (Ciuonzo et al., 2013), they provided a twofold generalization, allowing sensors to be non identical on one hand and introducing diversity on the other hand. Along with the derivation, we provide also a general tool to verify optimality of the received energy test in scenarios with correlated sensor decisions. Finally, we derive an analytical expression of the effect of the diversity on the large-system performances, under both individual and total power constraints. 


\subsection{Performance of Modulation Techniques}

The transmission from the base station to mobile or downlink transmission using 16-ary Quadrature Amplitude Modulation (QAM), 64-ary QAM and Quadrature Phase Shift Keying (QPSK) modulation schemes were considered in Code Division Multiple Access (CDMA) system. They had done the implementation and analysis of these modulation techniques when the system was subjected to Genetic Algorithm (GA) for multiuser detection and Additive White Gaussian Noise (AWGN) with fading and Rayleigh fading in the channel (Desai et al., 2012). The research has been performed by using simulation in MATLAB 7.7 and evaluation of Bit Error Rate (BER) against Signal-to-Noise Ratio (SNR) for CDMA system model. From analysis of three modulation techniques, the system could use more appropriate modulation technique to suit the channel quality, thus they could deliver the optimum and efficient data rate to mobile terminal.

\subsection{Energy Optimization}

The energy consumption for components of a typical sensor node and discuss the main directions to energy saving methods in wireless sensor networks. Then they presented a methodical and comprehensive taxonomy of the energy optimization methods in wireless sensor networks (Sachan et al., 2012). The main goal of energy optimization methods was to collect and aggregate data in an energy efficient manner so that network life time was enhanced. They concluded with possible future research directions.

\subsection{Speed Optimization}

The wireless communication has become quiet prevalent all around the world. Mobility and the elimination of a wired infrastructure, the key benefits of wireless operations, give users the flexibility to communicate from wherever they might be at any given time. In that study they had explained different wireless network standards (Khan and Khan, 2012), different modes of wireless networking, ways to optimize performance and speed of wireless network and the future of wireless network.

\subsection{Neighbor Node Discovery}

The detection of new links and nodes during initialization was discussed (Cohen and Kapchits,
2011), i.e., when the node was in Init state and their detection during normal operation, when the node was in Normal state. The former will be referred to as initial neighbor discovery whereas the latter will be referred to as continuous neighbor discovery, while previous works addressed initial neighbor discovery and continuous neighbor discovery as similar tasks (Ciuonzo et al., 2013), to be performed by the same scheme.

\subsection{Improving QoS}

In study (Bajaber and Awan, 2011; Chen et al., 2011), the analysis was based on the retrieving sensor data such that QoS requirements were satisfied was a challenging problem and has not been studied until recently in those papers. The general approach was to apply redundancy to satisfy the QoS requirement. In the study they were also interested in applying redundancy to satisfy application specified reliability and timeliness requirements for query-based WSNs.

In this study, the concepts appeared in related papers were consolidated and improve with more special features and propose a new method.

\section{MATERIALS AND METHODS}

\subsection{Proposed Method}

The aim of the paper is to propose a methodology for wireless communication to reduce the data loss and to improve the performance of it.

Wireless Network is the network that handles the client-server request-response without any wired connection and thus it enhances mobility and reliability. The client-server process is the process of transmitting and receiving the data to and from the server. In most cases, the capacity and speed of these two nodes may vary. In such case, the process of transmission and receiving may lead to loss some data, because the speed between the nodes does not match.

\subsection{Data Loss and its Cons}

The data to be transmitted can be partitioned into number of packets. The transmission of data can be confirmed by means of acknowledgement. Once the data packet has been transmitted, the node waits for getting the acknowledgement. Only upon receiving the acknowledgement, the node is ready to send next data packet. If no acknowledgement received from the receiver, then the sender waits for receiving the 
acknowledgement and thus the performance delay can occur.

The acknowledgement can be lost due to two reasons:

- Receiver not receiving the data packet and so no acknowledgement can be sent

- Receiver sent the acknowledgement and it may lose on the channel

The packet can be sent or received through the channel established between the client and the server, through the radio-magnetic waves. The former of missing acknowledgement is due to Data Loss and the later is due to collision occurred on the channel.

\subsection{Proposed Architecture}

To avoid these kinds of problem, the proposed architecture improve the performance of wireless network. In this proposed methodology, a new device has been implemented to handle the speed variance between the client and the server. The device is termed as Windows Management Instrumentation (WMI). This is used on the top of the TCP/IP layer to gather the client details. The details include the client's speed, capacity and so on. These details are used by the Network Provider to handle the process of transmission of data.

In Fig. 3, the network provider can able to know the client details prior to start the process of transmission, through WMI. Based upon these details, the sender can send the data packet to the receiver and waits for the acknowledgement. If the sender can able to transmit about $25 \mathrm{~Kb} / \mathrm{sec}$ but the receiver can able to receive only $15 \mathrm{~Kb} / \mathrm{sec}$, then, with the help of WMI, this is informed to the sender in prior and thus the sender reduces the data capacity to $15 \mathrm{~Kb} / \mathrm{sec}$ to avoid the data loss. Due to this kind of reduction, the data transmitting speed and performance of the network can also be increased and the receiver can able to receive the data properly.

In Fig. 4, the architecture shows how the WMI layer that exists between the client and the server through communication channel to perform the task. The WMI can also be implemented on UDP layer to control the network performance on it.

The client-server connected through the communication channel with the user-defined port number. In addition to the communication channel, the WMI layer also placed on it to control the client-server processing. The information transmitted from the node can be validated by the WMI Layer and then it transmits to the opponent node as shown in Fig. 5. If the layer founds that the data capacity exceeds the receiver capacity, then the layer retransmits to the sender to adjust the capacity. Thus the data loss can be reduced and the speed can be increased, by avoiding the collision. Also the performance of the network can be increased. The data loss can be measured as follows:

$$
\begin{aligned}
& \text { Data Loss }(\%)=\text { Node Capacity } \\
& *(\text { Total Packets / Packets Received }) * 100
\end{aligned}
$$

The process of the proposed work can be explained through the algorithm which is given below.

\subsection{Algorithm}

Start

Step-1: Establish Connection

Get the Port number from the user, pno

Connect the server with the client using pno

Step-2: WMI Details WMI analyze the channel Identify the client details and its capacity, ccap

Step-3: Data Transmission

Data transmits from server to client, data

Find the data capacity, dcap

If dcap $<=$ ccap then number,

Transmit the data to the client with port

pno

Else

with

Retransmit the data to server for adjustment

client capacity, ccap

End if

Step-4: Server Adjustment

Server receives the data, data

Client capacity, ccap also received.

Adjust the dcap to ccap

Go to step-3

Step-5: client Process

Client receives the data, data

Client send the acknowledgement, ack

Step-6: Next Transmission

If ack received then

Go to step-3

Else

Waits for sometime

End if

End 
Lavanya Sankara Narayanan and Murali Baskaran Venugopal / Journal of Computer Science 10 (3): 443-452, 2014

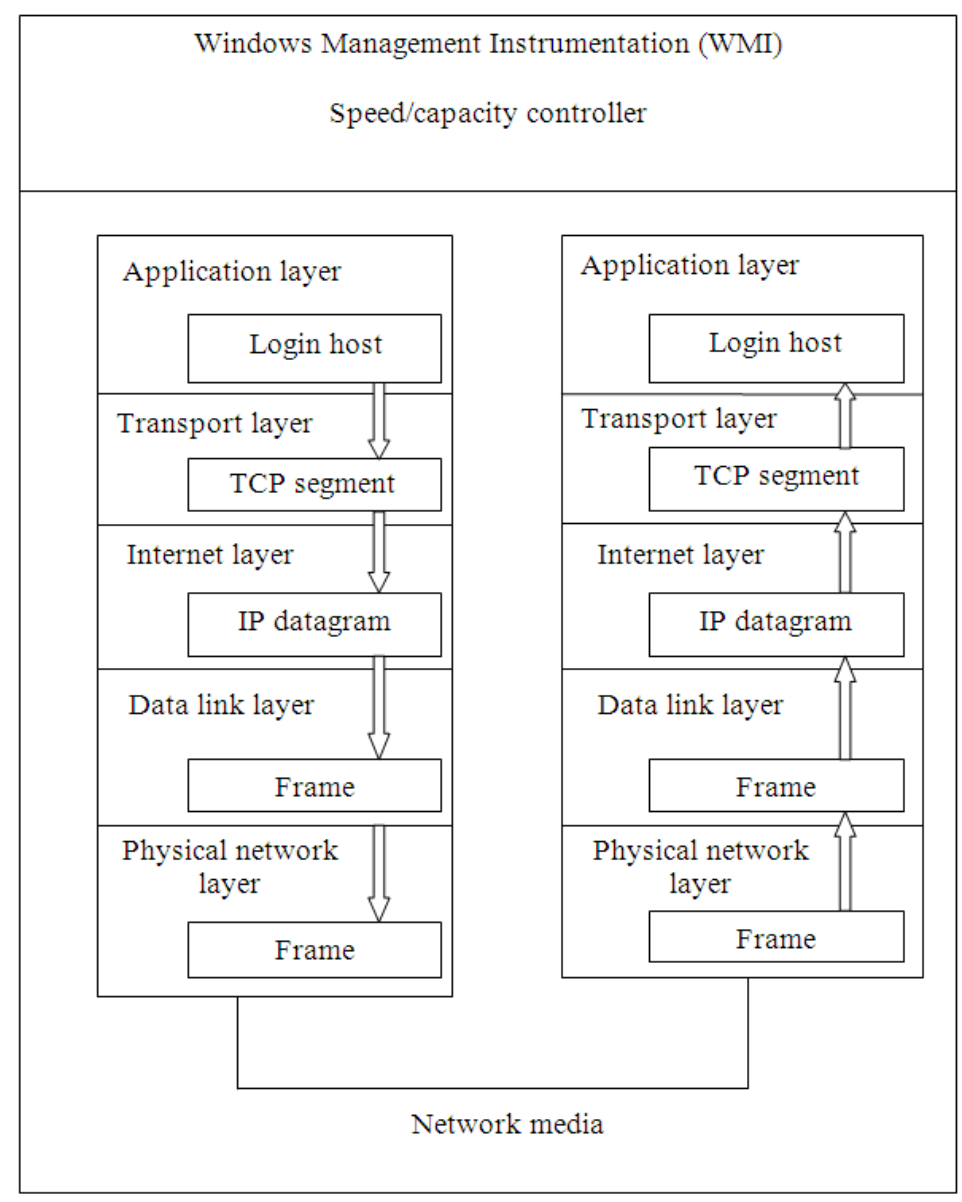

Fig. 3. WMI architecture

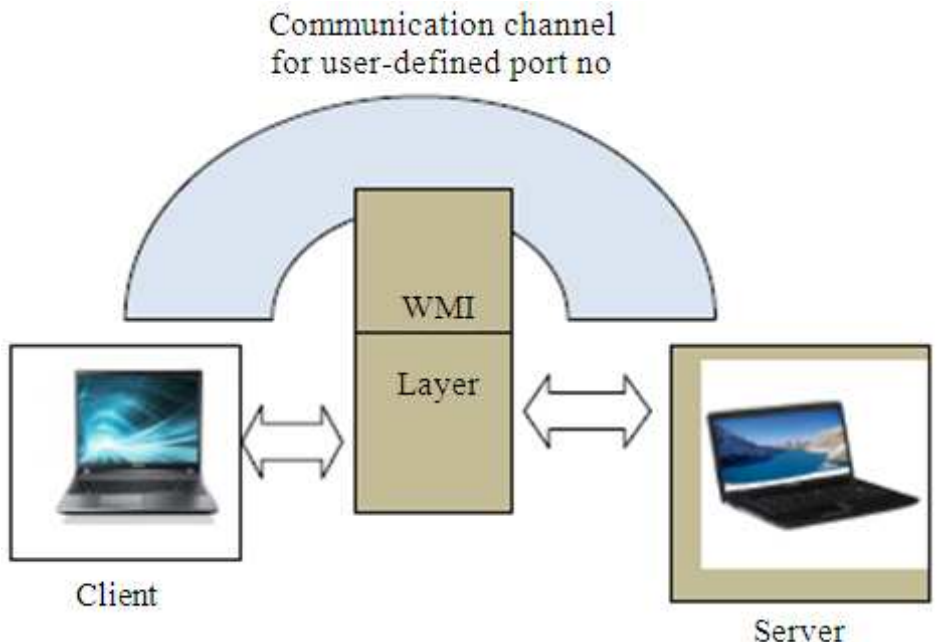

Fig. 4. Client-server architecture with WMI layer 


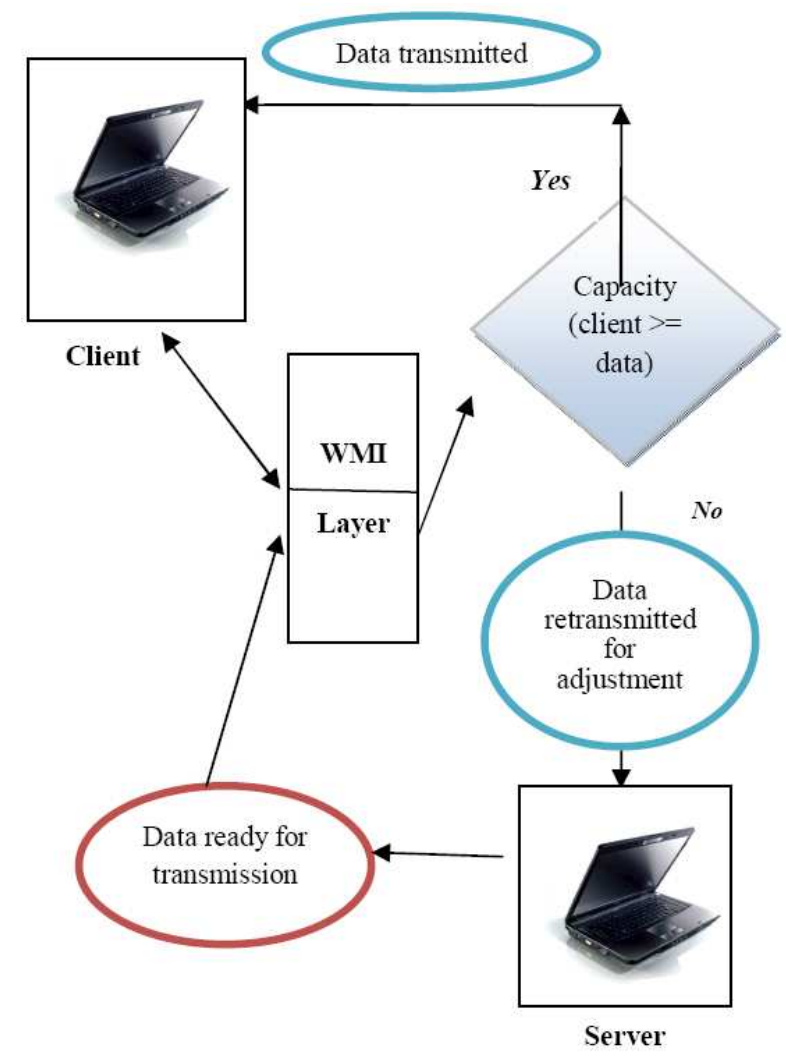

Fig. 5. Data transmission using WMI architecture

\section{RESULTS}

Various experiments can be undertaken to verify the proposed architecture that performs well than other existing methods such as piggyback method, continuous neighbor discovery and so on. In piggyback method, the packet loss can be controlled by encryption techniques, whereas there may be the possibility of packet loss due to speed variance. In continuous neighbor discovery, it is not sure to discover the neighbor in mobile environment. The experimental setup is made by comparing the private existing network with the proposed architecture. For this experiment, we use a service provider. Using this service provider, several users are called to transmit the data through both kinds of network and the result is verified through transmission time, data loss and so on. The proposed architecture reduces the data loss much and thus the performance of the network increases. The time taken to encrypt and decrypt the packet and to identify the network will be reduced through WMI layer. The performance rate is shown in the table below.
Table 1. Performance of network based on WMI architecture

\begin{tabular}{lll}
\hline $\begin{array}{l}\text { No. of } \\
\text { users }\end{array}$ & $\begin{array}{l}\text { Data loss (\%) using } \\
\text { existing networks }\end{array}$ & $\begin{array}{l}\text { Data loss (\%) using } \\
\text { proposed networks }\end{array}$ \\
\hline 100 & 5.0 & 0.001 \\
200 & 7.5 & 0.010 \\
300 & 8.0 & 0.100 \\
400 & 10.0 & 1.000 \\
500 & 20.0 & 1.010 \\
\hline
\end{tabular}

The Table 1, shows the variation in data loss by using the WMI layer in communication channels between the client and the server. Huge number of users may access the network using various channels and the data loss will occur due to transmission rate and collision and it will be reduced by the implementation of WMI layer shown in Fig. 6.

The above graph Fig. 6, shows the performance based on the number of users in the network during the communication process at a period of time. The existing system may have the data loss as high due to improper acknowledgement and while using the WMI layer the data loss will be reduced due to proper communication channel. 


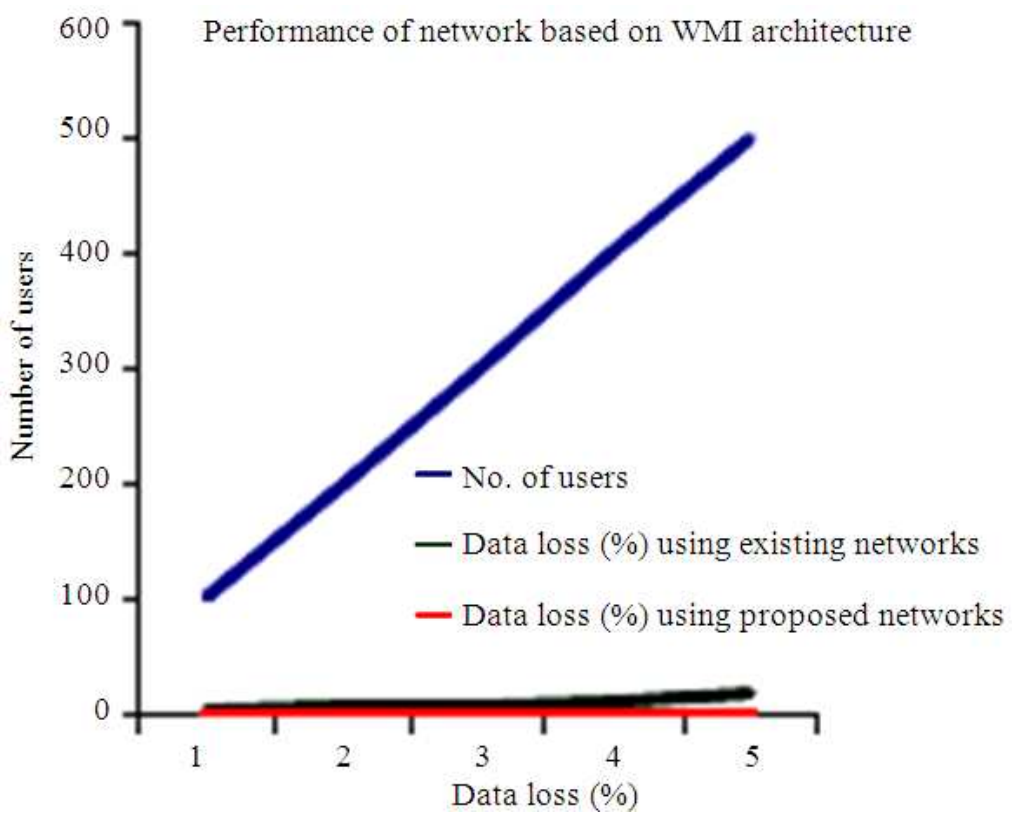

Fig. 6. Performance of network based on WMI Architecture

\section{DISCUSSION}

\subsection{Data Loss}

In wireless network, the data transmission between the client and server through multiple channel. In which, the acknowledgement may not be received by the client due to heavy data loss. Huge number of users transmit data to various clients at a time. Collision may occur due to network traffic. The network lifetime will also be more.

\subsection{WMI Layer}

The WMI layer was included in the OSI to control the speed of the transmission. The tranmission rate will be calculated based on the speed of the network.

The data transmission from server to client is based on the network capacity such as client $>=$ data. The WMI layer may transmitthe data from server through the network channels.

\subsection{Network Provider}

A Network Provider was introduced using WMI layer in our proposed system, for huge number of users in a network. The algorithm was developed based on client capacity and server adjustment to reduce the data loss during transmission in the network.

\subsection{Comparison: Two network are choosen}

one is the exisitng network and the other one is proposed network. The proposed network consist of the network provider and the data transmission is through WMI Layer. Both exisitng and proposed network consist of same number of users and data for the transmission as per Table 1. The comparison shows the improvement in data loss between same set of users.

\section{CONCLUSION}

Thus the proposed architecture helps to reduce the data rate and to improve the performance of the wireless network. It can be made possible by means WMI layer lies between the sender and the receiver to control the capacity of the nodes. If the data loss can be reduced, the collision also gets reduced. If both the data loss and the collision reduced, the performance of the network gets increased and this will improve the reliability of the network. Thus the proposed architecture performs well in any kinds of network and provides the user with better performance.

\section{ACKNOWLEDGEMENT}

We are thankful to the institution for making us able to do something. We express our gratitude to Prof.K. Selvi, Head of the Department of Information 
Technology, for suggesting the merging of the architecture to calculate transmission efficiency and wholehearted support for such activities. Finally, our acknowledgement cannot end without thanking the authors whose research papers helped us in developing this system.

\section{REFERENCES}

Bajaber, F. and I. Awan, 2011. Adaptive decentralized re-clustering protocol for wireless sensor networks. J. Comput. Syst. Sci., 77: 282-292. DOI: 10.1016/j.jcss.2010.01.007

Banavar, M.K., A.D. Smith, C. Tepedelenlioglu and A. Spanias, 2012. On the effectiveness of multiple antennas in distributed detection over fading MACs. IEEE Trans. Wireless Commun., 11: 1744-1752. DOI: 10.1109/TWC.2012.031212.110814

Chen, I.R., A.P. Speer and M. Eltoweissy, 2011. Adaptive fault-tolerant QoS control algorithms for maximizing system lifetime of query-based wireless sensor networks. IEEE Trans. Dependable Secure Comput., 8: 161-176. DOI: 10.1109/TDSC.2009.54

Ciuonzo, D., G. Romano and P.S. Rossi, 2012. Channelaware decision fusion in distributed MIMO wireless sensor networks: Decode-and-fuse vs. decode-thenfuse. IEEE Trans. Wireless Commun., 11: 29762985. DOI: 10.1109/TWC.2012.061912.112049

Ciuonzo, D., G. Romano and P.S. Rossi, 2013. Optimality of received energy in decision fusion over rayleigh fading diversity MAC with nonidentical sensors. IEEE Trans. Signal Process., 61: 22-27. DOI: 10.1109/TSP.2012.2223694
Cohen, R. and B. Kapchits, 2011. Continuous neighbor discovery in asynchronous sensor networks. IEEE/ACM Trans. Network., 19: 69-79. DOI: 10.1109/TNET.2010.2053943

Desai, J., K.G. Maradia and N.B. Gohil, 2012. Performance analysis of modulation techniques in GA assisted CDMA wireless communication system with AWGN Rayleigh fading channel. Int. J. Scient. Technol. Res., 1: 59-62. Dhandhukia, N. and K. Parmar, 2012. Effects of jamming attacks on wireless communication systems. Int. J. Sci. Eng. Res., 3: 1-6.

Khan, M.E. and F. Khan, 2012. An empirical study of different modes of wireless network communication and ways to optimize its performance and speed. Int. J. Comput. Applic., 46: 44-50. DOI: 10.5120/69649583

Merzoug, M.A. and A. Boukerram, 2011. Cluster-based communication protocol for load-balancing in wireless sensor networks. Int. J. Adv. Comput. Sci. Applic., 3: 105-112.

Sachan, V.K., S.A. Imam and J.M. Islamia, 2012. Energy-efficient communication methods in wireless sensor networks: A critical review. Int. J. Comput. Applic., 39: 35-48. DOI: 10.5120/49157484 